SLAC-TN-14-004

Replaces: SLAC CN-35

\title{
Parasitic Mode Losses in the Damping Ring
}

\author{
By: Perry Wilson \\ SLAC, National Accelerator Laboratory, Stanford, California 94309 USA
}

The two major sources of parasitic mode loss impedance in the damping ring will be the RF cavities (higher order modes) and the injection/ejection kickers. Treating the latter component first, we assume that the kicker chamber is a ceramic tube boated on the inside with kovar (1.3 ohms/square), as designed for CESR \$^1\$. 


\section{SINGLE PASS COL}

\section{TITLE: Parasitic Mode Losses in the Damping Ring}

The two major sources of parasitic mode loss impedance in the damping ring will be the RF cavities (higher order modes) and the injection/ejection kickers. Treating the latter component first, we assume that the kicker chamber is a ceramic tube coated on the inside with kovar ( 1.3 ohms/square), as designed for CESR $^{2}$. For a chamber of this type, it has been found that the energy loss per pulse is just the resistive loss given by

$$
W=R \int I^{2}(t) d t
$$

where $I(t)$ is the instantaneous current in the beam pulse and $R$ is the dc resistance of the coating, given by

$$
R=R_{S} L / 2 \pi a \text {. }
$$

Here $R_{S}$ is the surface resistance of the coating, $L$ is the length of the chamber and $a$ is its radius. Carrying out the integration for a gaussian pulse, the loss parameter becomes

$$
k=\frac{H}{q^{2}}=\frac{R}{2 \sqrt{\pi} \sigma_{t}} \text {. }
$$

Assuming $R_{s}=1.5$ ohms, $L=30 \mathrm{~cm}$ and $a=1 \mathrm{~cm}$, we compute $R=7 \Omega$. For $\sigma_{t}=17 \mathrm{ps}$ (corresponding to a damped bunch length of $5 \mathrm{~mm}$ ), $k=0.12$.

Turning to the RF cavities, we can estimate the loss parameter starting from computed values for higher modes in the PEP cavities. For example, the PEP cell with a $=3.8 \mathrm{~cm}$ has $k=1.2 \mathrm{~V} / \mathrm{pC}$ for $\sigma_{z}=0$. This scales to $2.4 \mathrm{~V} / \mathrm{pC}$ at $714 \mathrm{MHz}$, with $a=1.9 \mathrm{~cm}$. Using other cavity data, we estimate $k=2.0$ at $\sigma_{z}=1 \mathrm{~mm}$ and $k=1.0$ at $\sigma_{z}=5 \mathrm{~mm}$ for a cavity with $a=2.0 \mathrm{~cm}$ at $714 \mathrm{MHz}$. This variation of $k$ with $\sigma_{z}$ can be represented approximately by

$$
k / \text { cell }=2.4 \exp \left[-0.17 \sigma_{z}(\mathrm{~mm})\right] \quad \mathrm{V} / \mathrm{pC}
$$

1. R. Dixon, F. Messing, D. Morse and A. Sadoff, IEEE Trans. Nuct. Sci. NS-24, 1337 (1977) 
For four cells at $\sigma_{z}=5 \mathrm{~mm}, k=4.1 \mathrm{~V} / \mathrm{pC}$. Adding $k=0.24$ for two kickers and including $k=0.16$ for miscellaneous losses, we have a total loss parameter of $4.5 \mathrm{~V} / \mathrm{pC}$ for the damping ring at $\sigma_{z}=5 \mathrm{~mm}$. Multiplying by the time between bunches (55 ns) we obtain a loss impedance of $2.5 \times 10^{5}$ ohms. For a circulating current of $142 \mathrm{~mA}$, the energy loss per turn per particle is $35 \mathrm{keV}$. Values at other bunch lengths are readily computed from Eqs. (1) and (2). 